\title{
Some unified results on stochastic properties of residual lifetimes at random times
}

\author{
Neeraj Misra and Sameen Naqvi \\ Indian Institute of Technology Kanpur
}

\begin{abstract}
The residual life of a random variable $X$ at random time $\Theta$ is defined to be a random variable $X_{\Theta}$ having the same distribution as the conditional distribution of $X-\Theta$ given $X>\Theta$ (denoted by $X_{\Theta}=(X-\Theta \mid X>$ $\Theta)$ ). Let $\left(X, \Theta_{1}\right)$ and $\left(Y, \Theta_{2}\right)$ be two pairs of jointly distributed random variables, where $X$ and $\Theta_{1}$ (and, $Y$ and $\Theta_{2}$ ) are not necessarily independent. In this paper, we compare random variables $X_{\Theta_{1}}$ and $Y_{\Theta_{2}}$ by providing sufficient conditions under which $X_{\Theta_{1}}$ and $Y_{\Theta_{2}}$ are stochastically ordered with respect to various stochastic orderings. These comparisons have been made with respect to hazard rate, likelihood ratio and mean residual life orders. We also study various ageing properties of random variable $X_{\Theta_{1}}$. By considering this generalized model, we generalize and unify several results in the literature on stochastic properties of residual lifetimes at random times. Some examples to illustrate the application of the results derived in the paper are also presented.
\end{abstract}

\section{Introduction}

Let the lifetime of a component be represented by a non-negative random variable (r.v.) $X$ having absolutely continuous distribution function (d.f.) $F(\cdot)$, probability density function (p.d.f.) $f(\cdot)$ and survival function (s.f.) $\bar{F}(\cdot)=1-F(\cdot)$. Then, the residual life of the component which has survived up to time $t, t>0$, is given by r.v. $X_{t}$ having the same distribution as conditional distribution of $X-t$ given $X>t$ (denoted by $X_{t}=(X-t \mid X>t)$ ). If $t$ is replaced by a r.v. $\Theta$, then $X_{\Theta}=$ $(X-\Theta \mid X>\Theta)$ represents the residual lifetime of r.v. $X$ at random time $\Theta$. The following situations illustrate the interpretation of r.v. $X_{\Theta}$ :

- In clinical trials, it often happens that the time at which a person goes to clinic for examination of a disease is actually different from the time he got infected. In this scenario, the latent period of the disease can be estimated by $X_{\Theta}=(X-$ $\Theta \mid X>\Theta)$ (Cha and Finkelstein (2014), Finkelstein and Vaupel (2015)).

- Consider a series system with two components $C_{1}$ and $C_{2}$, having lifetimes $\Theta$ and $X$, respectively. If $C_{1}$ fails before $C_{2}$, then the system will fail to work but $C_{2}$ may still be in working condition. In this situation, $X_{\Theta}$ can be used to determine the residual life of $C_{2}$ after the failure of $C_{1}$.

Key words and phrases. Hazard rate order, likelihood ratio order, mean residual life order, reversed hazard rate order.

Received July 2016; accepted December 2016. 
The concept of residual life at a random time (RLRT), or at fixed time, has been studied and discussed extensively in the literature (Stoyan (1983), Guess and Proschan (1988), Shaked and Shanthikumar (2007), Cai and Zheng (2012)). Various researchers have presented results on stochastic comparisons of RLRT and have discussed its ageing properties (Yue and Cao (2000, 2001), Li and Zuo (2004), Misra, Gupta and Dhariyal (2008), Eryilmaz (2013), Dewan and Khaledi (2014)). These results have been derived either by assuming that $X \stackrel{d}{=} Y$ or $\Theta_{1} \stackrel{d}{=} \Theta_{2}$; here, $\stackrel{d}{=}$ means equality in distribution. Moreover, all the studies carried out so far have assumed that $X$ and $\Theta_{1}$ (and, $Y$ and $\Theta_{2}$ ) are independently distributed. Under the assumptions that $X \stackrel{d}{=} Y, X$ and $\Theta_{1}$, and $Y$ and $\Theta_{2}$ are independently distributed, the following results are available in the literature:

(i) Yue and Cao (2000) established that if $\Theta_{1} \leq \mathrm{rh} \Theta_{2}$ and $X$ has decreasing (increasing) failure rate, then $X_{\Theta_{1}} \leq_{\mathrm{st}}\left(\geq_{\mathrm{st}}\right) X_{\Theta_{2}}$. This result was further strengthened by Misra, Gupta and Dhariyal (2008) where, under the same assumptions as in Yue and Cao (2000), it is proved that $X_{\Theta_{1}} \leq_{\mathrm{hr}}\left(\geq_{\mathrm{hr}}\right) X_{\Theta_{2}}$. Recently, Dewan and Khaledi (2014) gave a different proof of this result of Misra, Gupta and Dhariyal (2008).

(ii) Under the assumptions that $\Theta_{1} \leq_{\mathrm{rh}} \Theta_{2}$ and $X$ has decreasing (increasing) mean residual life, Yue and Cao (2000) proved that $E\left(X_{\Theta_{1}}\right) \leq(\geq) E\left(X_{\Theta_{2}}\right)$. This result was generalized by $\mathrm{Li}$ and Zuo (2004) who, under the same assumptions as in Yue and Cao (2000), established increasing convex order between $X_{\Theta_{1}}$ and $X_{\Theta_{2}}$. Later on, Misra, Gupta and Dhariyal (2008), in their Theorem 3.2, further strengthened the result of Li and Zuo (2004) by establishing the mean residual life order between $X_{\Theta_{1}}$ and $X_{\Theta_{2}}$. Dewan and Khaledi (2014) in their Theorem 2.8 (d) provided an alternate proof of the above result proved by Misra, Gupta and Dhariyal (2008).

Dewan and Khaledi (2014) assumed that $\Theta_{1} \stackrel{d}{=} \Theta_{2}, X$ and $\Theta_{1}$, and $Y$ and $\Theta_{2}$ are independently distributed, and proved the following results:

(i) If $X \leq_{\mathrm{rh}} Y$ and either $X$ or $Y$ has increasing reversed failure rate, then $X_{\Theta} \leq$ rh $Y_{\Theta}$.

(ii) If $X \leq_{\mathrm{hr}} Y$ and either $X$ or $Y$ has decreasing failure rate, then $X_{\Theta} \leq_{\mathrm{hr}} Y_{\Theta}$.

(iii) If $X \leq_{\operatorname{mrl}} Y$ and either $X$ or $Y$ has increasing mean residual life, then $X_{\Theta} \leq \mathrm{mrl} Y_{\Theta}$.

The purpose of this study is to unify and generalize aforementioned results by considering stochastic comparison of $X_{\Theta_{1}}$ and $Y_{\Theta_{2}}$, without assuming that $X \stackrel{d}{=} Y$ or $\Theta_{1} \stackrel{d}{=} \Theta_{2}$, and also without assuming that $X$ and $\Theta_{1}$ (and, $Y$ and $\Theta_{2}$ ) are independently distributed. The general layout of the model considered in this paper is as follows: Let $X, Y, \Theta_{1}$, and $\Theta_{2}$ be non negative r.v.s with $\Theta_{i}, i=1,2$, having p.d.f. $h_{i}$, d.f. $H_{i}$ and s.f. $\bar{H}_{i}$. Let $\left(X, \Theta_{1}\right)$ and $\left(Y, \Theta_{2}\right)$ be two pairs of jointly 
distributed r.v.s with the common support $[0, \infty) \times[0, \infty)$. For any fixed $\theta>0$, let $X_{\theta}\left(Y_{\theta}\right)$ denote the r.v. having the same distribution as the conditional distribution of $X(Y)$ given that $\Theta_{1}=\theta\left(\Theta_{2}=\theta\right)$. Let $f_{\theta}(\cdot), F_{\theta}(\cdot), \bar{F}_{\theta}(\cdot)\left(g_{\theta}(\cdot), G_{\theta}(\cdot)\right.$, $\left.\bar{G}_{\theta}(\cdot)\right)$, respectively be the p.d.f., d.f. and s.f. of $X_{\theta}\left(Y_{\theta}\right), \theta>0$. The residual life of r.v. $X(Y)$ at random time $\Theta_{1}\left(\Theta_{2}\right)$ is given by $X_{\Theta_{1}}=\left(X-\Theta_{1} \mid X>\Theta_{1}\right)$ $\left(Y_{\Theta_{2}}=\left(Y-\Theta_{2} \mid Y>\Theta_{2}\right)\right)$. The s.f.s of $X_{\Theta_{1}}$ and $Y_{\Theta_{2}}$ are given by

$$
\begin{array}{ll}
\bar{M}_{1}(x)=\frac{\int_{0}^{\infty} \bar{F}_{\theta}(x+\theta) h_{1}(\theta) d \theta}{\int_{0}^{\infty} \bar{F}_{\theta}(\theta) h_{1}(\theta) d \theta}, & \text { if } x \geq 0, \quad \text { and } \\
\bar{M}_{2}(x)=\frac{\int_{0}^{\infty} \bar{G}_{\theta}(x+\theta) h_{2}(\theta) d \theta}{\int_{0}^{\infty} \bar{G}_{\theta}(\theta) h_{2}(\theta) d \theta}, & \text { if } x \geq 0,
\end{array}
$$

respectively. The density functions of $X_{\Theta_{1}}$ and $Y_{\Theta_{2}}$ are given by

$$
\begin{array}{ll}
m_{1}(x)=\frac{\int_{0}^{\infty} f_{\theta}(x+\theta) h_{1}(\theta) d \theta}{\int_{0}^{\infty} \bar{F}_{\theta}(\theta) h_{1}(\theta) d \theta}, & \text { if } x>0, \quad \text { and } \\
m_{2}(x)=\frac{\int_{0}^{\infty} g_{\theta}(x+\theta) h_{2}(\theta) d \theta}{\int_{0}^{\infty} \bar{G}_{\theta}(\theta) h_{2}(\theta) d \theta}, & \text { if } x>0,
\end{array}
$$

respectively.

Note that models (1.1) and (1.2) are conditionally dependent mixture models. Recently, various authors have studied stochastic properties of conditionally independent mixture models. Some of the contributions in this direction are due to Gupta, Dhariyal and Misra (2011), Gupta and Kirmani A (2006) and Misra, Gupta and Gupta (2009).

This paper is organized as follows: In Section 2, we mention some auxiliary results which will be used in proving the main results of the paper. Section 3 presents results on stochastic comparison of $X_{\Theta_{1}}$ and $Y_{\Theta_{2}}$ with respect to various stochastic orders. Specifically, we focus on stochastic comparisons with respect to hazard rate, likelihood ratio and mean residual life orders. In Section 4, we discuss ageing properties of random variable $X_{\Theta_{1}}$, and finally, in Section 5, we present some examples to illustrate applications of the results derived in the paper.

\section{Preliminaries}

Throughout the paper, the terms increasing and decreasing will imply nondecreasing and non-increasing, respectively. Consider a r.v. $X_{i}, i=1,2$, having absolutely continuous d.f. $F_{i}$, s.f. $\bar{F}_{i}$ and the Lebesgue p.d.f. $f_{i}, i=1,2$. Further, for the sake of simplicity, assume that distributions of $X_{1}$ and $X_{2}$ have the common support $[0, \infty)=\left\{t \in \mathbb{R}: f_{i}(t)>0\right\}, i=1,2$. For ease of reference, we first review some standard notations and definitions before stating our main results. We begin with definitions of some standard stochastic orders and ageing notions (see Shaked and Shanthikumar (2007), Lai and Xie (2006), Li and Li (2013)). 
Definition 2.1. The r.v. $X_{1}$ is said to be smaller than the r.v. $X_{2}$ in

(i) hazard rate order (denoted by $\left.X_{1} \leq_{\mathrm{hr}} X_{2}\right)$ if $\bar{F}_{2}(x) / \bar{F}_{1}(x)$ increases in $x>0$;

(ii) reversed hazard rate order (denoted by $\left.X_{1} \leq_{\mathrm{rh}} X_{2}\right)$ if $F_{2}(x) / F_{1}(x)$ increases in $x>0$;

(iii) likelihood ratio order (denoted by $\left.X_{1} \leq_{\operatorname{lr}} X_{2}\right)$ if $f_{2}(x) / f_{1}(x)$ increases in $x>0$;

(iv) mean residual life order (denoted by $X_{1} \leq \operatorname{mrl} X_{2}$ ) if $\int_{x}^{\infty} \bar{F}_{2}(u) d u /$ $\int_{x}^{\infty} \bar{F}_{1}(u) d u$ increases in $x>0$.

Definition 2.2. The r.v. $X$ is said to have the

(i) increasing (decreasing) likelihood ratio (ILR (DLR)) if $f(x)$ is logconcave (log-convex) on $(0, \infty)$;

(ii) increasing (decreasing) failure rate (IFR (DFR)) if $\bar{F}(x)$ is log-concave (log-convex) on $(0, \infty)$;

(iii) decreasing reversed failure rate (DRFR) if $F(x)$ is log-concave on $(0, \infty)$;

(iv) increasing (decreasing) mean residual life (IMRL (DMRL)) if $\int_{x}^{\infty} \bar{F}(t) d t$ is log-convex (log-concave) on $(0, \infty)$.

The next definition on totally positive and reverse regular functions may be found in Karlin (1968).

Definition 2.3. Let $S_{1}, S_{2} \subseteq \mathbb{R}$ and let $k: S_{1} \times S_{2} \rightarrow[0, \infty)$ be a non-negative function, where $\mathbb{R}$ denotes the real line. The function $k(x, y)$ is said to be totally positive (reverse regular) of order 2 , denoted by $\mathrm{TP}_{2}\left(\mathrm{RR}_{2}\right)$, if $k\left(x_{1}, y_{1}\right) k\left(x_{2}, y_{2}\right) \geq$ $(\leq) k\left(x_{1}, y_{2}\right) k\left(x_{2}, y_{1}\right)$, whenever $x_{1} \leq x_{2}, y_{1} \leq y_{2}, x_{1}, x_{2} \in S_{1}$ and $y_{1}, y_{2} \in S_{2}$.

Dewan and Khaledi (2014) and Khaledi (2014) have listed a few results due to Karlin (1968) and Joag-Dev, Kochar and Proschan (1995) on $T P_{2}\left(R R_{2}\right)$ functions. We now state the following lemma, proved in Naqvi (2017), which will be helpful in deriving the main results of this paper and may also be of independent interest to researchers. This lemma extends the results mentioned in Dewan and Khaledi (2014) (also see Khaledi (2014) and Misra and van der Meulen (2003)).

Let $\psi_{i}:[0, \infty) \times[0, \infty) \rightarrow \mathbb{R}, i=1,2$, be a function and let $g_{i}(\theta)$ be the Lebesgue p.d.f. of a r.v. $T_{i}, i=1,2$. In many branches of statistics, one often encounters the problem of verifying the monotonicity of function of the type

$$
\psi(x)=\frac{\int_{0}^{\infty} \psi_{2}(x, \theta) g_{2}(\theta) d \theta}{\int_{0}^{\infty} \psi_{1}(x, \theta) g_{1}(\theta) d \theta}, \quad x>0 .
$$

In the following lemma, we provide sufficient conditions on $\psi_{i}(\cdot, \cdot), i=1,2$, for the function in (2.1) to be monotone. 
Lemma 2.4. Suppose that $\frac{\psi_{2}(x, \theta)}{\psi_{1}(x, \theta)}$ increases (decreases) in $x \in(0, \infty)$ and increases in $\theta \in(0, \infty)$. Further suppose that any of the following three conditions hold:

(i) $T_{1} \leq_{\operatorname{lr}} T_{2}$ and $\psi_{1}(x, \theta)$ or $\psi_{2}(x, \theta)$ is $T P_{2}\left(R R_{2}\right)$ in $(x, \theta) \in(0, \infty) \times$ $(0, \infty)$

(ii) $T_{1} \leq \mathrm{hr} T_{2}$ and $\psi_{1}(x, \theta)$ is $T P_{2}\left(R R_{2}\right)$ in $(x, \theta) \in(0, \infty) \times(0, \infty)$ and is increasing in $\theta \in(0, \infty)$ or $\psi_{2}(x, \theta)$ is $T P_{2}\left(R R_{2}\right)$ in $(x, \theta) \in(0, \infty) \times(0, \infty)$ and is increasing in $\theta \in(0, \infty)$

(iii) $T_{1} \leq \mathrm{rh} T_{2}$ and $\psi_{1}(x, \theta)$ is $T P_{2}\left(R R_{2}\right)$ in $(x, \theta) \in(0, \infty) \times(0, \infty)$ and is decreasing in $\theta \in(0, \infty)$ or, $\psi_{2}(x, \theta)$ is $T P_{2}\left(R R_{2}\right)$ in $(x, \theta) \in(0, \infty) \times(0, \infty)$ and is decreasing in $\theta \in(0, \infty)$.

Then, the function $\psi(x)$, as defined in (2.1), increases (decreases) in $x \in(0, \infty)$.

\section{Main results}

In this section, we carry out stochastic comparisons of residual lifetimes at random times. The first result presents sufficient conditions for stochastic monotonicity in terms of the likelihood ratio order.

Theorem 3.1. Suppose that $\Theta_{1} \leq_{\operatorname{lr}} \Theta_{2}$ and that the following assumptions are fulfilled:

(i) $f_{\theta}(x+\theta)$ or $g_{\theta}(x+\theta)$ is $T P_{2}\left(R R_{2}\right)$ in $(x, \theta) \in(0, \infty) \times(0, \infty)$;

(ii) For every fixed $\theta>0, g_{\theta}(x+\theta) / f_{\theta}(x+\theta)$ is increasing (decreasing) in $x \in(0, \infty)$;

(iii) For every fixed $x>0, g_{\theta}(x+\theta) / f_{\theta}(x+\theta)$ is increasing in $\theta \in(0, \infty)$.

Then, $X_{\Theta_{1}} \leq_{\mathrm{lr}}\left(\geq_{\mathrm{lr}}\right) Y_{\Theta_{2}}$.

Proof. From (1.2), it suffices to prove that

$$
\psi_{1}^{*}(x)=\frac{m_{2}(x)}{m_{1}(x)}=\frac{\int_{0}^{\infty} g_{\theta}(x+\theta) h_{2}(\theta) d \theta}{\int_{0}^{\infty} f_{\theta}(x+\theta) h_{1}(\theta) d \theta}
$$

is increasing (decreasing) in $x \in(0, \infty)$. Define $\psi_{1}(x, \theta)=f_{\theta}(x+\theta)$ and $\psi_{2}(x, \theta)=g_{\theta}(x+\theta),(x, \theta) \in(0, \infty) \times(0, \infty)$. Now, upon applying Lemma 2.4(i) with $T_{1} \stackrel{d}{=} \Theta_{1}$ and $T_{2} \stackrel{d}{=} \Theta_{2}$, it can be proved that the function $\psi_{1}^{*}(x)$ increases (decreases) in $x \in(0, \infty)$. Hence the theorem follows. 
Remark 3.2. It is useful to observe that condition (i) of Theorem 3.1, which is equivalent to saying that $f_{\theta_{2}}\left(x_{2}+\theta_{2}\right) f_{\theta_{1}}\left(x_{1}+\theta_{1}\right) \geq(\leq) f_{\theta_{2}}\left(x_{1}+\theta_{2}\right) f_{\theta_{1}}\left(x_{2}+\theta_{1}\right)$, for $0<x_{1}<x_{2}<\infty$ and $0<\theta_{1}<\theta_{2}<\infty$ is satisfied if $X_{\theta}$ has DLR (ILR) and $X_{\theta_{1}} \leq_{\text {lr }}\left(\geq_{\text {lr }}\right) X_{\theta_{2}}, \forall 0<\theta_{1} \leq \theta_{2}$. Also, it can be easily seen that condition (ii) of Theorem 3.1 holds if $X_{\theta} \leq_{\operatorname{lr}}\left(\geq_{\operatorname{lr}}\right) Y_{\theta}, \forall \theta>0$.

The above remark leads to the following corollary.

Corollary 3.3. Suppose that $\Theta_{1} \leq_{1 \mathrm{r}} \Theta_{2}$ and that the following assumptions are fulfilled:

(i) For every $\theta>0, X_{\theta}$ has $\operatorname{DLR}\left(\right.$ ILR) and $X_{\theta_{1}} \leq_{\mathrm{lr}}\left(\geq_{\mathrm{lr}}\right) X_{\theta_{2}}, \forall 0<\theta_{1} \leq \theta_{2}$; or,

For every $\theta>0, Y_{\theta}$ has $D L R(I L R)$ and $Y_{\theta_{1}} \leq_{\operatorname{lr}}\left(\geq_{\mathrm{lr}}\right) Y_{\theta_{2}}, \forall 0<\theta_{1} \leq \theta_{2}$;

(ii) $X_{\theta} \leq \operatorname{lr}(\geq \operatorname{lr}) Y_{\theta}, \forall \theta>0$;

(iii) Condition (iii) of Theorem 3.1 holds.

Then, $X_{\Theta_{1}} \leq_{\text {lr }}\left(\geq_{\text {lr }}\right) Y_{\Theta_{2}}$.

As a consequence of Theorem 3.1, we immediately obtain the following corollary, which compares the residual lifetimes at random times in terms of the likelihood ratio order for the case when $X_{\theta}$ and $Y_{\theta}$ are identically distributed.

Corollary 3.4. Assume that $X_{\theta} \stackrel{d}{=} Y_{\theta}, \forall \theta>0, f_{\theta}(x+\theta)$ is $T P_{2}\left(R R_{2}\right)$ in $(x, \theta) \in$ $(0, \infty) \times(0, \infty)$, and $\Theta_{1} \leq_{\text {lr }} \Theta_{2}$. Then, $X_{\Theta_{1}} \leq_{\text {lr }}\left(\geq_{\text {lr }}\right) X_{\Theta_{2}}$.

It is to be noted here that when $X$ and $\Theta_{1}$ are independently distributed, and $Y$ and $\Theta_{2}$ are independently distributed, we have $X_{\theta} \stackrel{d}{=} X$ and $Y_{\theta} \stackrel{d}{=} Y$, i.e., $f_{\theta} \equiv f$ and $g_{\theta} \equiv g, \theta>0$. In addition, if $X \stackrel{d}{=} Y$, then $f \equiv g$. Thus, we have the following corollary to Theorem 3.1.

Corollary 3.5. Let $X$ and $\Theta_{1}$ be independently distributed, and let $Y$ and $\Theta_{2}$ be also independently distributed.

(i) If $X$ or $Y$ has DLR, $X \leq \leq_{l r} Y$, and $\Theta_{1} \leq_{l r} \Theta_{2}$, then $X_{\Theta_{1}} \leq_{l r} Y_{\Theta_{2}}$.

(ii) Let $X \stackrel{d}{=} Y$. If $X$ has DLR (ILR), and $\Theta_{1} \leq l r \Theta_{2}$, then $X_{\Theta_{1}} \leq l r\left(\geq_{l r}\right) X_{\Theta_{2}}$.

(iii) Let $\Theta_{1} \stackrel{d}{=} \Theta_{2}$. If $X$ or $Y$ has $D L R$, and $X \leq l r Y$, then $X_{\Theta} \leq l r Y_{\Theta}$.

Remark 3.6. It is worth mentioning here that there are typos in Theorem 2.2(a) and Theorem 2.8(a) of Dewan and Khaledi (2014). In these theorems, ILR should be replaced by DLR, and vice-versa. Under this correction, Corollary 3.5(ii) and (iii) above are the corresponding versions of Theorem 2.2(a) and Theorem 2.8(a) of Dewan and Khaledi (2014). 
In what follows, we would like to compare two residual lifetimes with respect to hazard rate ordering. In the following theorem, likelihood ratio order is assumed between $\Theta_{1}$ and $\Theta_{2}$ and sufficient conditions are obtained to establish hazard rate ordering between $X_{\Theta_{1}}$ and $Y_{\Theta_{2}}$. The proof follows on using Lemma 2.4(i) with $T_{1} \stackrel{d}{=} \Theta_{1}, T_{2} \stackrel{d}{=} \Theta_{2}, \psi_{1}(x, \theta)=\bar{F}_{\theta}(x+\theta)$ and $\psi_{2}(x, \theta)=\bar{G}_{\theta}(x+\theta),(x, \theta) \in$ $(0, \infty) \times(0, \infty)$.

Theorem 3.7. Suppose that $\Theta_{1} \leq_{l r} \Theta_{2}$ and that the following assumptions are fulfilled:

(i) $\bar{F}_{\theta}(x+\theta)$ or $\bar{G}_{\theta}(x+\theta)$ is $T P_{2}\left(R R_{2}\right)$ in $(x, \theta) \in(0, \infty) \times(0, \infty)$;

(ii) For every fixed $\theta>0, \bar{G}_{\theta}(x+\theta) / \bar{F}_{\theta}(x+\theta)$ is increasing (decreasing) in $x \in(0, \infty)$

(iii) For every fixed $x>0, \bar{G}_{\theta}(x+\theta) / \bar{F}_{\theta}(x+\theta)$ is increasing in $\theta \in(0, \infty)$.

Then, $X_{\Theta_{1}} \leq \mathrm{hr}\left(\geq_{\mathrm{hr}}\right) Y_{\Theta_{2}}$.

Remark 3.8. It needs to be mentioned that condition (i) of Theorem 3.7 is satisfied if, $\forall \theta>0, X_{\theta}$ has DFR (IFR) and $X_{\theta_{1}} \leq_{\mathrm{hr}}\left(\geq_{\mathrm{hr}}\right) X_{\theta_{2}}, \forall 0<\theta_{1} \leq \theta_{2}$. Also, it can be easily seen that condition (ii) of Theorem 3.7 holds if $X_{\theta} \leq_{\mathrm{hr}}\left(\geq_{\mathrm{hr}}\right) Y_{\theta}, \forall \theta>0$. As a consequence, we obtain the following result for hazard rate ordering between $X_{\Theta_{1}}$ and $Y_{\Theta_{2}}$.

Corollary 3.9. Suppose that $\Theta_{1} \leq_{l r} \Theta_{2}$ and that the following assumptions are fulfilled:

(i) For every $\theta>0, X_{\theta}$ has DFR (IFR) and $X_{\theta_{1}} \leq_{\mathrm{hr}}\left(\geq_{\mathrm{hr}}\right) X_{\theta_{2}}, \forall 0<\theta_{1} \leq \theta_{2}$ or,

For every $\theta>0, Y_{\theta}$ has DFR (IFR) and $Y_{\theta_{1}} \leq \mathrm{hr}\left(\geq_{\mathrm{hr}}\right) Y_{\theta_{2}}, \forall 0<\theta_{1} \leq \theta_{2}$;

(ii) $X_{\theta} \leq_{\mathrm{hr}}\left(\geq_{\mathrm{hr}}\right) Y_{\theta}, \forall \theta>0$;

(iii) Condition (iii) of Theorem 3.7 holds.

Then, $X_{\Theta_{1}} \leq \mathrm{hr}\left(\geq_{\mathrm{hr}}\right) Y_{\Theta_{2}}$.

It is of interest to know whether conclusions of Theorem 3.7 may still hold if $\Theta_{1}$ and $\Theta_{2}$ are ordered with respect to hazard rate or reversed hazard rate order. The following theorem gives an answer.

Theorem 3.10. Suppose that conditions (ii) and (iii) of Theorem 3.7 hold true. In addition, suppose that either of the following two assumptions are fulfilled:

(i) $\Theta_{1} \leq_{\mathrm{hr}} \Theta_{2}$ and

$\bar{F}_{\theta}(x+\theta)$ is $T P_{2}\left(R R_{2}\right)$ in $(x, \theta) \in(0, \infty) \times(0, \infty)$ and is increasing in $\theta \in(0, \infty)$ or

$\bar{G}_{\theta}(x+\theta)$ is $T P_{2}\left(R R_{2}\right)$ in $(x, \theta) \in(0, \infty) \times(0, \infty)$ and is increasing in $\theta \in(0, \infty)$; 
(ii) $\Theta_{1} \leq_{r h} \Theta_{2}$ and $\bar{F}_{\theta}(x+\theta)$ is $T P_{2}\left(R R_{2}\right)$ in $(x, \theta) \in(0, \infty) \times(0, \infty)$ and is decreasing in $\theta \in(0, \infty)$ or, $\bar{G}_{\theta}(x+\theta)$ is $T P_{2}\left(R R_{2}\right)$ in $(x, \theta) \in(0, \infty) \times(0, \infty)$ and is decreasing in $\theta \in(0, \infty)$.

Then, $X_{\Theta_{1}} \leq_{\mathrm{hr}}\left(\geq_{\mathrm{hr}}\right) Y_{\Theta_{2}}$.

Remark 3.11. It is useful to observe that when the assumption of stronger stochastic order between $\Theta_{1}$ and $\Theta_{2}$ (i.e., $\Theta_{1} \leq_{l r} \Theta_{2}$ ) in Theorem 3.7 is replaced by a weaker stochastic ordering $\left(\Theta_{1} \leq_{\mathrm{hr}}\left(\leq_{r h}\right) \Theta_{2}\right)$ in Theorem 3.10, then an extra condition on $\bar{F}_{\theta}(x+\theta)$ or $\bar{G}_{\theta}(x+\theta)$ is required for the same result to hold. Thus, there exists a trade-off between these two set of conditions.

The following corollary, which readily follows from Theorem 3.7 and Theorem 3.10, explains how one can compare $X_{\Theta_{1}}$ and $X_{\Theta_{2}}$ in terms of hazard rate ordering based on the likelihood ratio, hazard rate and reversed hazard order between $\Theta_{1}$ and $\Theta_{2}$.

Corollary 3.12. Assume that $X_{\theta} \stackrel{d}{=} Y_{\theta}, \forall \theta>0$. Further suppose that either of the following three assumptions are fulfilled:

(i) $\Theta_{1} \leq l r \Theta_{2}$ and $\bar{F}_{\theta}(x+\theta)$ is $T P_{2}\left(R R_{2}\right)$ in $(x, \theta) \in(0, \infty) \times(0, \infty)$;

(ii) $\Theta_{1} \leq_{\mathrm{hr}} \Theta_{2}$ and $\bar{F}_{\theta}(x+\theta)$ is $T P_{2}\left(R R_{2}\right)$ in $(x, \theta) \in(0, \infty) \times(0, \infty)$ and is increasing in $\theta \in(0, \infty)$;

(iii) $\Theta_{1} \leq_{r h} \Theta_{2}$ and $\bar{F}_{\theta}(x+\theta)$ is $T P_{2}\left(R R_{2}\right)$ in $(x, \theta) \in(0, \infty) \times(0, \infty)$ and is decreasing in $\theta \in(0, \infty)$.

Then, $X_{\Theta_{1}} \leq_{\mathrm{hr}}\left(\geq_{\mathrm{hr}}\right) X_{\Theta_{2}}$.

As an immediate consequence of Theorem 3.10(ii), we have the following corollary.

Corollary 3.13. Assume that $X$ and $\Theta_{1}$ are independently distributed, and $Y$ and $\Theta_{2}$ are also independently distributed.

(i) If $X$ or $Y$ has DFR, $X \leq_{\mathrm{hr}} Y$, and $\Theta_{1} \leq_{r h} \Theta_{2}$, then $X_{\Theta_{1}} \leq_{\mathrm{hr}} X_{\Theta_{2}}$.

(ii) Let $X \stackrel{d}{=} Y$. If $X$ has DFR $(I F R)$, and $\Theta_{1} \leq_{r h} \Theta_{2}$, then $X_{\Theta_{1}} \leq \mathrm{hr}\left(\geq_{\mathrm{hr}}\right) X_{\Theta_{2}}$.

(iii) Let $\Theta_{1} \stackrel{d}{=} \Theta_{2}$. If $X$ or $Y$ has DFR, and $X \leq \mathrm{hr} Y$, then $X_{\Theta} \leq \mathrm{hr} Y_{\Theta}$.

Remark 3.14. Misra, Gupta and Dhariyal (2008) and Dewan and Khaledi (2014) presented the result of Corollary 3.13(ii) as Theorem 3.1 and Theorem 2.8(c), respectively. Dewan and Khaledi (2014) also reported the result of Corollary 3.13(iii) as Theorem 2.2 (c). 
Now, we provide results concerning comparison of residual lifetimes with respect to mean residual life order. The following result follows on using Lemma 2.4(i) with $T_{1} \stackrel{d}{=} \Theta_{1}, T_{2} \stackrel{d}{=} \Theta_{2}, \psi_{1}(x, \theta)=\int_{x}^{\infty} \bar{F}_{\theta}(u+\theta) d u$ and $\psi_{2}(x, \theta)=$ $\int_{x}^{\infty} \bar{G}_{\theta}(u+\theta) d u,(x, \theta) \in(0, \infty) \times(0, \infty)$.

Theorem 3.15. Suppose that $\Theta_{1} \leq_{l r} \Theta_{2}$ and that the following assumptions hold: $(0, \infty)$

(i) $\int_{x}^{\infty} \bar{F}_{\theta}(u+\theta) d u$ or $\int_{x}^{\infty} \bar{G}_{\theta}(u+\theta) d u$ is $T P_{2}\left(R R_{2}\right)$ in $(x, \theta) \in(0, \infty) \times$

(ii) For every fixed $\theta>0, \int_{x}^{\infty} \bar{G}_{\theta}(u+\theta) d u / \int_{x}^{\infty} \bar{F}_{\theta}(u+\theta) d u$ is increasing (decreasing) in $x \in(0, \infty)$;

(iii) For every fixed $x>0, \int_{x}^{\infty} \bar{G}_{\theta}(u+\theta) d u / \int_{x}^{\infty} \bar{F}_{\theta}(u+\theta) d u$ is increasing in $\theta \in(0, \infty)$.

Then, $X_{\Theta_{1}} \leq_{m r l}\left(\geq_{m r l}\right) Y_{\Theta_{2}}$.

Remark 3.16. It should be noted here that condition (i) holds true if $X_{\theta}$ has $I M R L$ $(D M R L), \forall \theta>0$ and $X_{\theta_{1}} \leq_{m r l}\left(\geq_{m r l}\right) X_{\theta_{2}}, \forall 0<\theta_{1} \leq \theta_{2}$. In addition, it can be readily seen that condition (ii) is satisfied if $X_{\theta} \leq_{m r l}\left(\geq_{m r l}\right) Y_{\theta}, \forall \theta>0$. As a consequence, we obtain the following corollary to Theorem 3.15.

Corollary 3.17. Suppose that $\Theta_{1} \leq_{l r} \Theta_{2}$ and that the following assumptions hold:

(i) For every $\theta>0, X_{\theta}$ has IMRL $(D M R L)$ and $X_{\theta_{1}} \leq_{m r l}\left(\geq_{m r l}\right) X_{\theta_{2}}, \forall 0<$ $\theta_{1} \leq \theta_{2}$ or

For every $\theta>0, Y_{\theta}$ has IMRL $(D M R L)$ and $Y_{\theta_{1}} \leq_{m r l}\left(\geq_{m r l}\right) Y_{\theta_{2}}, \forall 0<$ $\theta_{1} \leq \theta_{2}$

(ii) $X_{\theta} \leq_{m r l}\left(\geq_{m r l}\right) Y_{\theta}, \forall \theta>0$;

(iii) condition (iii) of Theorem 3.15 holds.

Then, $X_{\Theta_{1}} \leq_{m r l}\left(\geq_{m r l}\right) Y_{\Theta_{2}}$.

The following theorem, provides some sufficient conditions for mean residual life order between $X_{\Theta_{1}}$ and $Y_{\Theta_{2}}$ under the assumption of hazard rate (reversed hazard rate) order between $\Theta_{1}$ and $\Theta_{2}$

Theorem 3.18. Suppose that conditions (ii) and (iii) of Theorem 3.15 hold true. In addition, suppose that either of the following two assumptions are fulfilled:

(i) $\Theta_{1} \leq_{\mathrm{hr}} \Theta_{2}$ and

$\int_{x}^{\infty} \bar{F}_{\theta}(u+\theta) d u$ is $T P_{2}\left(R R_{2}\right)$ in $(x, \theta) \in(0, \infty) \times(0, \infty)$ and is increasing in $\theta \in(0, \infty)$ or,

$\int_{x}^{\infty} \bar{G}_{\theta}(u+\theta) d u$ is $T P_{2}\left(R R_{2}\right)$ in $(x, \theta) \in(0, \infty) \times(0, \infty)$ and is increasing in $\theta \in(0, \infty)$; 
(ii) $\Theta_{1} \leq_{r h} \Theta_{2}$ and

$\int_{x}^{\infty} \bar{F}_{\theta}(u+\theta) d u$ is $T P_{2}\left(R R_{2}\right)$ in $(x, \theta) \in(0, \infty) \times(0, \infty)$ and is decreasing in $\theta \in(0, \infty)$ or,

$\int_{x}^{\infty} \bar{G}_{\theta}(u+\theta) d u$ is $T P_{2}\left(R R_{2}\right)$ in $(x, \theta) \in(0, \infty) \times(0, \infty)$ and is decreasing in $\theta \in(0, \infty)$.

Then, $X_{\Theta_{1}} \leq_{m r l}\left(\geq_{m r l}\right) Y_{\Theta_{2}}$.

Proof. The proof of the theorem follows from Lemma 2.4(ii) and (iii) with $T_{1} \stackrel{d}{=} \Theta_{1}, T_{2} \stackrel{d}{=} \Theta_{2}, \psi_{1}(x, \theta)=\int_{x}^{\infty} \bar{F}_{\theta}(u+\theta) d u$ and $\psi_{2}(x, \theta)=\int_{x}^{\infty} \bar{G}_{\theta}(u+\theta) d u$, $(x, \theta) \in(0, \infty) \times(0, \infty)$.

Remark 3.19. As discussed in Remark 3.11, there exists a trade-off between conditions of Theorem 3.15 and Theorem 3.18.

Upon applying an argument similar to that used in Theorem 3.15 and Theorem 3.18, an analogue of Corollary 3.12 on the mean residual life order can also be established.

Corollary 3.20. Assume that $X_{\theta} \stackrel{d}{=} Y_{\theta}, \forall \theta>0$. Further, suppose that either of the following three assumptions are fulfilled:

(i) $\Theta_{1} \leq_{l r} \Theta_{2}$ and $\int_{x}^{\infty} \bar{F}_{\theta}(u+\theta) d u$ is $T P_{2}\left(R R_{2}\right)$ in $(x, \theta) \in(0, \infty) \times$ $(0, \infty)$;

(ii) $\Theta_{1} \leq_{\mathrm{hr}} \Theta_{2}$ and $\int_{x}^{\infty} \bar{F}_{\theta}(u+\theta) d u$ is $T P_{2}\left(R R_{2}\right)$ in $(x, \theta) \in(0, \infty) \times(0, \infty)$ and is increasing in $\theta \in(0, \infty)$;

(iii) $\Theta_{1} \leq_{r h} \Theta_{2}$ and $\int_{x}^{\infty} \bar{F}_{\theta}(u+\theta) d u$ is $T P_{2}\left(R R_{2}\right)$ in $(x, \theta) \in(0, \infty) \times(0, \infty)$ and is decreasing in $\theta \in(0, \infty)$.

Then, $X_{\Theta_{1}} \leq_{m r l}\left(\geq_{m r l}\right) X_{\Theta_{2}}$.

As an immediate consequence of Theorem 3.18(ii), we have the following corollary.

Corollary 3.21. Assume that $X$ and $\Theta_{1}$ are independently distributed, and that $Y$ and $\Theta_{2}$ are also independently distributed.

(i) If $X$ or $Y$ has IMRL, $X \leq{ }_{m r l} Y$, and $\Theta_{1} \leq_{r h} \Theta_{2}$, then $X_{\Theta_{1}} \leq m r l Y_{\Theta_{2}}$.

(ii) Let $X \stackrel{d}{=} Y$. If $X$ has IMRL $(D M R L)$, and $\Theta_{1} \leq_{r h} \Theta_{2}$, then $X_{\Theta_{1}} \leq m r l$ $\left(\geq_{m r l}\right) X_{\Theta_{2}}$.

(iii) Let $\Theta_{1} \stackrel{d}{=} \Theta_{2}$. If $X$ or $Y$ has IMRL, and $X \leq{ }_{m r l} Y$, then $X_{\Theta} \leq{ }_{m r l} Y_{\Theta}$.

Remark 3.22. Misra, Gupta and Dhariyal (2008) and Dewan and Khaledi (2014) obtained the result of Corollary 3.21(ii) as Theorem 3.2 and Theorem 2.8(d), respectively. Dewan and Khaledi (2014) also presented the result of Corollary 3.21(iii) as Theorem 2.2(d). 


\section{Ageing properties}

In this section, we investigate under what conditions the ageing property of $X_{\theta}$ is preserved for $X_{\Theta_{1}}$. In the first place, we discuss a result for $D F R$ property of $X_{\Theta_{1}}$.

Theorem 4.1. Suppose that, for every fixed $x>0$ and $t>0, \frac{\bar{F}_{\theta}(\theta+x+t)}{\bar{F}_{\theta}(\theta+x)}$ increases in $\theta \in(0, \infty), X_{\theta}$ has DFR (IFR), $\forall \theta>0$, and $X_{\theta_{1}} \leq_{\mathrm{hr}}\left(\mathrm{Z}_{\mathrm{hr}}\right) X_{\theta_{2}}, \forall 0<\theta_{1} \leq \theta_{2}$. Then $X_{\Theta_{1}}$ has DFR (IFR).

Proof. To prove $X_{\Theta_{1}}$ has $D F R$ (IFR), we need to show that $\bar{M}_{1}(x)$ is log-convex (log-concave) on $(0, \infty)$, i.e., for every fixed $t>0, \frac{\bar{M}_{1}(x+t)}{\bar{M}_{1}(x)}$ increases (decreases) in $x \in(0, \infty)$. Consider

$$
\psi_{2}(x)=\frac{\bar{M}_{1}(x+t)}{\bar{M}_{1}(x)}=\frac{\int_{0}^{\infty} \bar{F}_{\theta}(\theta+x+t) h_{1}(\theta) d \theta}{\int_{0}^{\infty} \bar{F}_{\theta}(\theta+x) h_{1}(\theta) d \theta}, \quad x \in(0, \infty) .
$$

Let $\psi_{1}(x, \theta)=\bar{F}_{\theta}(\theta+x)$ and $\psi_{2}(x, \theta)=\bar{F}_{\theta}(\theta+x+t),(x, \theta) \in(0, \infty) \times(0, \infty)$, it can be easily observed that, for every $\theta>0, \frac{\psi_{2}(x, \theta)}{\psi_{1}(x, \theta)}=\frac{\bar{F}_{\theta}(\theta+x+t)}{\bar{F}_{\theta}(\theta+x)}$ increases (decreases) in $x \in(0, \infty)$, if $X_{\theta}$ has $D F R$ (IFR). Also, it can be easily shown that $\bar{F}_{\theta}(\theta+x)$ is $T P_{2}\left(R R_{2}\right)$ in $(x, \theta) \in(0, \infty) \times(0, \infty)$ if $X_{\theta}$ has $D F R(I F R)$, and $X_{\theta_{1}} \leq_{\mathrm{hr}}\left(\geq_{\mathrm{hr}}\right) X_{\theta_{2}}, \forall 0<\theta_{1} \leq \theta_{2}$. From these observations, we can conclude that the assumptions of Lemma 2.4(i) are satisfied under the assumptions of Theorem 4.1. Hence, $\psi_{2}(x)$ is increasing (decreasing) in $x \in(0, \infty)$.

As a consequence, we can obtain the following result immediately on assuming independence between $X$ and $\Theta_{1}$ in Theorem 4.1.

Corollary 4.2. Assume that $X$ and $\Theta_{1}$ are independently distributed and $X$ has DFR. Then $X_{\Theta_{1}}$ has DFR.

Remark 4.3. Under the assumption of independence between $X$ and $\Theta_{1}$, Yue and Cao (2000) in their Theorem 4.1 showed that, if $\Theta_{1}$ has DRFR and $X$ has DFR $(I F R)$, then $X_{\Theta_{1}}$ also has $D F R(I F R)$. It is apparent that the result of Corollary 4.2 requires no condition on $\Theta_{1}$.

In what follows, we will present the result on ageing notion of $X_{\Theta_{1}}$ in terms of mean residual life function. Applying Lemma 2.4(i) with $\psi_{1}(x, \theta)=\int_{x}^{\infty} \bar{F}_{\theta}(u+$ $\theta) d u$ and $\psi_{2}(x, \theta)=\int_{x+t}^{\infty} \bar{F}_{\theta}(u+\theta) d u,(x, \theta) \in(0, \infty) \times(0, \infty)$, and employing arguments similar to that of Theorem 4.1, we have the following result.

Theorem 4.4. Suppose that, for every fixed $x>0$ and $t>0, \frac{\int_{x+t}^{\infty} \bar{F}_{\theta}(u+\theta) d u}{\int_{x}^{\infty} \bar{F}_{\theta}(u+\theta) d u}$ increases in $\theta \in(0, \infty), X_{\theta}$ has IMRL (DMRL), $\forall \theta>0$ and $X_{\theta_{1}} \leq{ }_{m r l}\left(\geq_{m r l}\right) X_{\theta_{2}}$, $\forall 0<\theta_{1} \leq \theta_{2}$. Then $X_{\Theta_{1}}$ has IMRL $(D M R L)$. 
Yue and Cao (2000) assumed that $X$ and $\Theta_{1}$ are independently distributed, and showed that if $\Theta_{1}$ has $D R F R$ and $X$ has $D M R L(I M R L)$, then $X_{\Theta_{1}}$ also has $D M R L$ (IMRL). In the following corollary, which follows from Theorems 4.4, we obtain the same result without imposing any condition on $\Theta_{1}$.

Corollary 4.5. Assume that $X$ and $\Theta_{1}$ are independently distributed and $X$ has IMRL. Then, $X_{\Theta_{1}}$ has IMRL.

\section{Examples}

To illustrate the usefulness of results derived in this paper, we present below examples that cannot be dealt with existing results in the literature, but where our results can be applied.

Example 5.1. For $\theta>0$, let the r.v.s $X_{\theta}$ and $Y_{\theta}$ follow Gamma distributions with p.d.f.s,

$$
f_{\theta}(x)= \begin{cases}\frac{\left(1+\theta^{2}\right)^{\alpha}}{\theta^{\alpha} \Gamma \alpha} \mathrm{e}^{-\left(\frac{1+\theta^{2}}{\theta}\right) x} x^{\alpha-1}, & \text { if } x>0, \\ 0, & \text { otherwise }\end{cases}
$$

and

$$
g_{\theta}(x)= \begin{cases}\frac{1}{\theta^{\alpha} \Gamma \alpha} \mathrm{e}^{-\frac{x}{\theta}} x^{\alpha-1}, & \text { if } x>0, \\ 0, & \text { otherwise }\end{cases}
$$

respectively, where $\alpha \in(0,1)$ is a fixed shape parameter. For $0<x_{1} \leq x_{2}<\infty$,

$$
\frac{g_{\theta}\left(x_{2}+\theta\right)}{g_{\theta}\left(x_{1}+\theta\right)}=\mathrm{e}^{-\left(x_{2}-x_{1}\right) \frac{c}{\theta}}\left(\frac{x_{2}+\theta}{x_{1}+\theta}\right)^{\alpha-1}
$$

increases in $\theta>0$. Thus, $g_{\theta}(x+\theta)$ is $T P_{2}$ in $(x, \theta) \in(0, \infty) \times(0, \infty)$. Also, note that for $\theta>0$,

$$
\frac{g_{\theta}(x+\theta)}{f_{\theta}(x+\theta)}=\frac{1}{\left(1+\theta^{2}\right)^{\alpha}} \mathrm{e}^{\theta(x+\theta)}
$$

increases in $x>0$ and $\theta>0$. Hence, the conditions of Theorem 3.1 are satisfied for any pair of r.v.s $\Theta_{1}$ and $\Theta_{2}$ such that $\Theta_{1} \leq l r \Theta_{2}$.

Example 5.2. Suppose that the joint density of $\left(X, \Theta_{1}\right)$ is

$$
g_{1}(x, \theta)= \begin{cases}\frac{1}{\theta^{\alpha}} \frac{1}{\Gamma \alpha \Gamma \beta_{1}} \mathrm{e}^{-\left(\frac{x}{\theta}+\theta\right)} x^{\alpha-1} \theta^{\beta_{1}-1}, & \text { if } x>0, \\ 0, & \text { otherwise }\end{cases}
$$


and the joint density of $\left(Y, \Theta_{2}\right)$ is

$$
g_{2}(x, \theta)= \begin{cases}\frac{1}{\theta^{\alpha}} \frac{1}{\Gamma \alpha \Gamma \beta_{2}} \mathrm{e}^{-\left(\frac{x}{\theta}+\theta\right)} x^{\alpha-1} \theta^{\beta_{2}-1}, & \text { if } x>0, \\ 0, & \text { otherwise }\end{cases}
$$

where $\alpha \in(0,1)$ and $0<\beta_{1}<\beta_{2}<\infty$ are fixed shape parameters. Clearly, for every $\theta>0, X_{\theta} \stackrel{d}{=} Y_{\theta}, X_{\theta}$ has p.d.f.

$$
f_{\theta}(x)=\frac{1}{\theta^{\alpha} \Gamma \alpha} \mathrm{e}^{-\frac{x}{\theta}} x^{\alpha-1}, x>0
$$

and p.d.f. of r.v. $\Theta_{i}, i=1,2$ is

$$
g_{i}(\theta)=\frac{1}{\Gamma \beta_{i}} \mathrm{e}^{-\theta} \theta^{\beta_{i}-1}, \theta>0 .
$$

It can be seen that for $0<x_{1} \leq x_{2}<\infty$,

$$
\frac{f_{\theta}\left(x_{2}+\theta\right)}{f_{\theta}\left(x_{1}+\theta\right)}=\mathrm{e}^{\left(\frac{x_{1}-x_{2}}{\theta}\right)}\left(\frac{x_{2}+\theta}{x_{1}+\theta}\right)^{\alpha-1}
$$

increases in $\theta \in(0, \infty), \forall \alpha \in(0,1)$. Also,

$$
\frac{h_{2}(\theta)}{h_{1}(\theta)}=\frac{\Gamma \beta_{1}}{\Gamma \beta_{2}} \theta^{\left(\beta_{2}-\beta_{1}\right)}
$$

increases in $\theta>0, \forall \beta_{2}>\beta_{1}$. Thus, $\Theta_{1} \leq_{l r} \Theta_{2}$ and conditions of Corollary 3.4 are satisfied.

Now, we state the following example to illustrate applications of Corollary 3.12.

\section{Example 5.3.}

(i) For $\theta>0$, let the random variable $X_{\theta}$ follow Exponential Distribution with survival function

$$
\bar{F}_{\theta}(x)=e^{-\frac{x}{\theta}}, x>0
$$

It can be easily seen that $\bar{F}_{\theta}(x+\theta)$ is $T P_{2}$ in $(x, \theta) \in(0, \infty) \times(0, \infty)$ and is also increasing in $\theta>0$. Thus, the conditions of Corollary 3.12(ii) are satisfied for any pair if r.v.s $\Theta_{1}$ and $\Theta_{2}$ such that $\Theta_{1} \leq$ hr $\Theta_{2}$.

(ii) For $\theta>0$, let the random variable $X_{\theta}$ follow Exponential Distribution with survival function

$$
\bar{F}_{\theta}(x)=e^{-\theta x}, x>0
$$

It can be easily seen that $\bar{F}_{\theta}(x+\theta)$ is $R R_{2}$ in $(x, \theta) \in(0, \infty) \times(0, \infty)$ and is also decreasing in $\theta>0$. Thus, the conditions of Corollary 3.12(iii) are satisfied for any pair if r.v.s $\Theta_{1}$ and $\Theta_{2}$ such that $\Theta_{1} \leq_{r h} \Theta_{2}$. 


\section{Acknowledgments}

Authors are indebted to the anonymous referee for the insightful comments and suggestions, which resulted in an improvement in the presentation of this manuscript. Sameen Naqvi's work was supported by Indian Institute of Technology Kanpur, India.

\section{References}

Cai, N. and Zheng, Y. (2012). Preservation of generalized ageing class on the residual life at random time. Journal of Statistical Planning and Inference 142, 148-154. MR2827137

Cha, J. H. and Finkelstein, M. (2014). Some notes on the unobserved parameters (frailties) in reliability modeling. Reliability Engineering and System Safety 123, 99-103.

Dewan, I. and Khaledi, B. E. (2014). On stochastic comparisons of residual life time at random time. Statistics and Probability Letters 88, 73-79. MR3178335

Eryilmaz, S. (2013). On residual lifetime of coherent systems after the $r$ th failure. Statistical Papers 54, 243-250. MR3016965

Finkelstein, M. and Vaupel, J. W. (2015). On random age and remaining lifetime for population of items. Applied Stochastic Models in Business and Industry 31, 681-689. MR3407893

Guess, F. and Proschan, F. (1988). 12 mean residual life: Theory and applications. Handbook of Statistics 7, 215-224.

Gupta, N., Dhariyal, I. D. and Misra, N. (2011). Reliability under random operating environment: Frailty models. Journal of Combinatorics, Information and System Sciences 36(1-4), 117.

Gupta, R. C. and Kirmani A, S. N. U. (2006). Stochastic comparisons in frailty models. Journal of Statistical Planning and Inference 136(10), 3647-3658. MR2256280

Joag-Dev, K., Kochar, S. and Proschan, F. (1995). A general composition theorem and its applications to certain partial orderings of distributions. Statistics and Probability Letters 22, 111-119. MR1327736

Karlin, S. (1968). Total Positivity 1. California: Stanford University Press. MR0230102

Khaledi, B. E. (2014). Karlin's basic composition theorems and stochastic orderings. Journal of the Iranian Statistical Society 13, 177-186. MR3293124

Lai, C. D. and Xie, M. (2006). Stochastic Ageing and Dependence for Reliability. Berlin: Springer. MR2223811

Li, X. and Zuo, M. J. (2004). Stochastic comparisons of residual life and inactivity time at a random time. Stochastic Models 20(2), 229-235. MR2048254

$\mathrm{Li}, \mathrm{H}$. and Li, X. (2013). Stochastic orders in reliability and risk. In Honor of Professor Moshe Shaked. Berlin: Springer. MR3157470

Misra, N., Gupta, N. and Gupta, R. D. (2009). Stochastic comparisons of multivariate frailty models. Journal of Statistical Planning and Inference 139(6), 2084-2090. MR2497562

Misra, N., Gupta, N. and Dhariyal, I. D. (2008). Stochastic properties of residual life and inactivity time at random time. Stochastic Models 24(1), 89-102. MR2384692

Misra, N. and van der Meulen, E. C. (2003). On stochastic properties of m-spacings. Journal of Statistical Planning and Inference 115(2), 683-697. MR1985890

Naqvi, S. (2017). A study on stochastic properties of mixture models. Ph.D. Thesis, Indian Institute of Technology Kanpur, Kanpur, India.

Shaked, M. and Shanthikumar, G. J. (2007). Stochastic Orders and Their Applications. New York: Springer. MR2265633 
Stoyan, D. (1983). Comparison Methods for Queues and Other Stochastic Models. New York: Wiley. MR0754339

Yue, D. and Cao, J. (2000). Some results on the residual life at random time. Acta Mathematicae Applicatea Sinica 16, 436-443. MR1807174

Yue, D. and Cao, J. (2001). The NBUL class of life distribution and replacement policy comparisons. Naval Research Logistics 48(7), 578-591. MR1853512

Department of Mathematics and Statistics Indian Institute of Technology Kanpur Kanpur 208016

India

E-mail: neeraj@iitk.ac.in nsameen@iitk.ac.in 